\title{
Chemical structure and the changing concept of vitamin A activity
}

\author{
By G. A. J. PITT, Department of Biochemistry, University of Liverpool, PO \\ Box 147, Liverpool L69 ${ }_{3} B X$
}

Studies on chemical structure and vitamin activity have an archaic tang, redolent of the pioneering days of vitamin research, and interest in them has long waned for most vitamins. Vitamin $A$ is an exception, partly because its molecular mode of action (apart from in the visual pigments) is uncertain, but largely because the concept of vitamin $A$ activity has not remained static.

The traditional method used to establish the structural requirements for a molecule to show vitamin $A$ activity was the rat growth promotion test: the restoration of growth in animals that have stopped gaining weight on an $A$ deficient diet.

From such procedures it was concluded that only limited variation was possible on the retinol molecule (Fig. $\mathrm{I}, \mathrm{R}=\mathrm{CH}_{2} \mathrm{OH}$ ) if the substance was to manifest more than nugatory vitamin $A$ activity. Some changes were possible at the end of the chain; for example retinoic acid (Fig. I, $\mathbf{R}=\mathrm{COOH}$ ) had growth-promoting activity. Modifications elsewhere in the molecule usually abolished activity or reduced it to a negligible amount (Ames, 1965 ; Pitt, 1965 ). The only exceptions to this rule were the 3 -dehydro (vitamin $A_{2}$ ) series of compounds, with an extra double bond between carbon atoms 3 and 4 in the ring, which had a third to a half of the activity of the corresponding vitamin $A_{1}$ compounds (see references in Pitt, 1965 ).

The concept of vitamin A activity was extended with the discovery by Dowling \& Wald (1960) that retinoic acid, although it discharged the systemic function of vitamin A, i.e. it maintained life, growth and general health, could not be reduced to retinaldehyde and thus could not maintain the visual pigments. Thompson $e t$ al. (1964) subsequently showed that retinoic acid would not serve in reproduction in rats.

This roughly describes the position when reviewed (Pitt, 1965) in the Nutrition Society's last symposium on vitamin A.

The topic was reanimated by the work of Sporn's group on the effect of compounds related to retinol in mouse epidermal cell cultures and in reversing

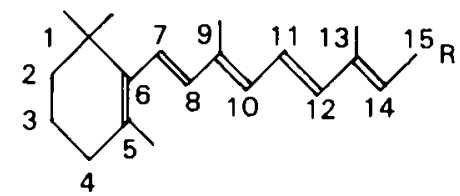

Fig. I. Retinol ( $\left.\mathrm{R} \cdot \mathrm{CH}_{2} \mathrm{OH}\right)$ with numbering system. 
vitamin A deficiency in hamster tracheal organ cultures (Clamon et al. 1974; Sporn et al. 1975; Sporn, Dunlop, Newton \& Henderson, 1976; Newton et al. I 980). These studies revealed that the structural specificity for a molecule to show vitamin A activity in cell or organ culture was very much less restricted than it had appeared to be in rat growth tests. Indeed the specificity was so wide and some of the compounds so different from retinol that Sporn, Dunlop, Newton \& Smith (1976) coined the term 'retinoid' to cover this broad family.

Some retinoids show substantial vitamin $A$ activity in vitro, but negligible activity in the rat growth test, e.g. an analogue of retinoic acid containing a dimethylacetylcyclopentenyl instead of a trimethylcyclohexenyl ring (Goodman et al. 1974; Sporn et al. 1975).

Another compound showing different activities in vitro and in the rat growthtest is $\alpha$-retinol (Fig. 2) (Pitt, 1969; Goodman et al. 1974; Sneider et al. 1974; Clamon et al. 1975; Sporn et al. 1975). Part of the discrepancy can be ascribed to the inability of $\alpha$-retinol to combine with plasma retinol-binding protein (RBP), the carrier necessary for the proper transport of vitamin A to target tissues. It consequently shows much poorer growth-promoting activity than retinol when given to animals, but as both can be delivered equally well into cultures, the difference there greatly diminishes. It seems that rat growth bioassays test, among other things, the structural specificity of plasma RBP (Willetts et al. 1976; Pitt, 1978).

The visual pigments provide another example of how the specificity of plasma RBP can influence the handling of retinoids and thus the manifestation of their biological activity in the whole animal.

Visual pigments consist of a protein of the opsin family joined with retinaldehyde (Fig. I, $\mathrm{R}=\mathrm{CHO}$ ). The specificity of opsins is quite wide; numerous modified retinaldehydes can form visual pigments in vitro (see, for example, Blatz et al. 1969; Morton \& Pitt, 1969, and other references given by Crouch et al. I981). Animals given these retinoids orally, however, do not usually form the expected pigments in their eyes (Morton \& Pitt, 1969). For example, aretinaldehyde, the aldehyde of $\alpha$-retinol, will unite with opsin in vitro to form an artificial ' $\alpha$-rhodopsin' (Houghton, 1969; Morton \& Pitt, 1969) but the administration of $\alpha$-retinol to rats does not result in the formation of a visual pigment (Sneider et al. 1974). This is presumably because combination with plasma RBP is necessary for uptake from the blood by the receptor sites in the pigment epithelium of the eye (Chen \& Heller, 1977), and indeed other target tissues (Bhat \& Cama, 1979; Rask et al. 1980, 1981).

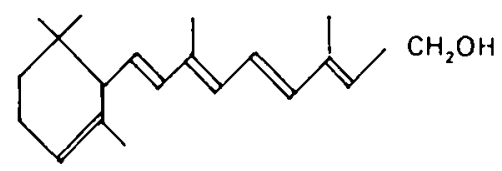

Fig. 2. $\alpha$-Retinol. 
Although the reports of Goodman \& Raz (1972), Horwitz \& Heller (1973, 1974) and Cogan et al. (1976) indicated that plasma RBP is capable of binding a number of compounds, a careful study by Muhilal \& Glover (1975) of the rates at which they combine pointed towards plasma RBPs operating rather more specifically. Even so, these studies in vitro probably indicate a less narrow specificity than prevails in vivo. Retinoic acid will join fairly readily with plasma RBP in vitro but in the animal it is carried by albumin not plasma RBP (Smith et al. 1973).

The inability of some retinoids to bind to plasma RBP is only one factor accounting for differences between activities determined in vitro and in vivo. Probably a more important one is the rapid destruction by the animal body of most retinoids other than retinol and 3 -dehydroretinol-a-retinol is one of the exceptions in being biologically fairly stable. Carboxylic acids are catabolized faster than their corresponding alcohols. This accounts for the different values obtained in vivo and in vitro for the potency of retinoic acid, which has variable activity in growth-promotion tests, but is always less effective than retinol, because it is eliminated from the body so rapidly (Zile \& DeLuca, I 968 ). In contrast, retinoic acid is consistently more effective in vitro than retinol (see Newton et al. 1980), presumably because in these conditions its slower destruction enables its potentiality to be expressed more fully. Similar considerations must apply to many retinoids. Absorbability, metabolism, tissue distribution and pharmacokinetics will vary between retinoids and result in different activities (Lotan et al. 1980; Trown et al. 1980).

Within cells are found two more binding proteins, cellular retinol-binding protein (CRBP)—different from plasma RBP - and cellular retinoic acid-binding protein (CRABP) (Chytil \& Ong, 1978). Their specificities are wider than that of plasma RBP (Chytil \& Ong, 1978; Lotan et al. 1980; Trown et al. 1980). While they roughly correspond to those for observable activities of retinoids (Lotan et al. 1980), there are differences; for example, retinoid carboxylic acids that have good biological activity always bind well to CRABP, but the converse is not true (Trown et al. 1980 ).

The precision of some of these in vitro studies (Sporn et al. 1975; Sporn, Dunlop, Newton \& Henderson, I976; Newton et al. I980; Trown et al. 1980) is uncertain, but a major advantage is that they work over a much wider range and at much lower concentrations than do the whole animal assays. For that reason they are able to reveal low-level activity in a compound that would not be measurable in a rat growth bioassay.

Hardly any of the retinoids found to be active in vitro but not in vivo occurred naturally; none was better than retinol or retinoic acid in curing vitamin $A$ deficiency; there seems no strictly nutritional advantage to be gained from these findings. What they have done, however, is to open up wide fields of research on two aspects of vitamin $A$ that had long been of some clinical interest: its use in the prevention of tumour growth and in dermatology.

From its known ability to control epithelial cell differentiation, vitamin A appeared of potential value in influencing the formation of carcinomas. As 
keratinization is a classic sign of vitamin A deficiency and a reversal of keratinization a sign of excess vitamin $A$, there were therapeutic possibilities in the treatment of skin disorders involving keratinization. Exploration of such activities of vitamin $A$ had been inhibited by the impression that one had to have retinol, or something very close to it, for substantial vitamin $A$ activity. The recognition that considerable variation was possible in the molecule when operating at the cellular level prompted the synthesis of a large number of retinoids with the specific intention of obtaining some showing more strongly than retinol characteristics useful for therapeutic purposes. Very large numbers of retinoids have been made and tested, most of them derivatives of retinoic acid, which has a short half-life in the body and, unlike retinol, is not accumulated in the liver.

Many retinoids, varying considerably in structure, have been tested for tumour prevention (see Bertram, 1980; Lotan et al. 1980; Newton et al. 1980; Trown et al. 1980). This is a wide and active field (see Lotan, 1980) and is being reviewed in this symposium (Hicks, 1983 ).

There has been considerable success in the dermatological work, particularly in the treatment of acne and psoriasis. Again the structural specificity for effective action is wide. Consider two compounds that have been valuable therapeutically. The ${ }_{13}$-cis isomer of retinoic acid has proved very useful in the treatment of acne (Peck et al. 1979). An aromatic retinoid made by Hoffmann-La Roche \& Co, Basle, as RO 10-9359 (Fig. 3) and marketed as etretinate or Tigason ${ }^{2}$ has been very effective for psoriasis (Tsambaos \& Orfanos, 1981 ). It is not known why such compounds have differential effects, clinically and also in experimental animals (Peck, I980; Gomez, 1981).

The major handicap to the therapeutic use of retinoids is their toxicity (Sporn, Dunlop, Newton \& Smith, 1976; Bollag \& Matter, 1981; Tsambaos \& Orfanos, 1981; Hicks, 1983). The activities of compounds in inducing hypervitaminosis $\mathrm{A}$ do not always parallel those in growth promotion. Notably, retinoic acid has lower growth-promoting activity than retinol, as mentioned, but induces hypervitaminosis A much more effectively (Harris, 1960; Thompson \& Pitt, 1960).

Physiological activity and toxicity sometimes do not compare well even in tests in vitro (Sporn, Dunlop, Newton \& Henderson, 1976). Since the desideratum is a compound showing the required biological activity with minimal toxic effects, the chemical specificity for hypervitaminosis $\mathrm{A}$ has been explored in various ways; for

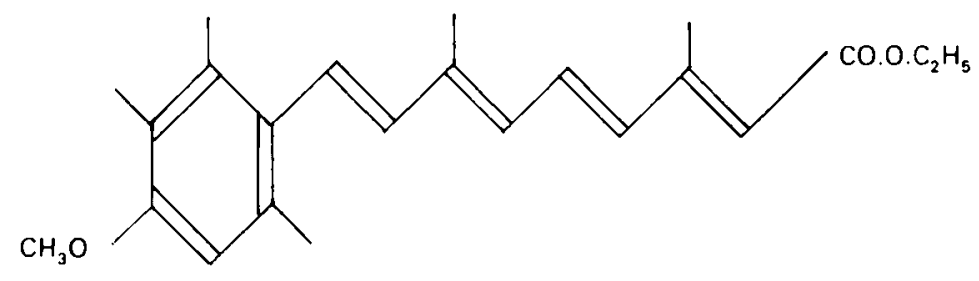

Fig. 3. RO 10-9359, an aromatic retinoid made by Hoffmann-La Roche \& Co, Basle. 
example in mice by using weight loss, desquamation of the skin, hair loss and bone fractures (Bollag \& Matter, 1981 ), in rats by measuring skeletal development (Turton et al. 1983) and also in organ culture (Goodman et al. 1974; Sporn, Dunlop, Newton \& Henderson, 1976; Bertram, 1980). Tests in vitro are quicker and cheaper but are not necessarily reliable indications of the effects on intact animals.

Another method of measuring the hypervitaminotic activity of compounds was developed some years ago at Liverpool. Hughes (1975) devised a bioassay procedure based on the finding (Morgan et al. 1969; Thompson et al. 1969) that very small amounts of retinoic acid, when injected into hens' eggs, are lethal to the early embryo. The $\mathrm{LD}_{30}$ can be determined, with a measure of its precision. Some values found were (means with $95 \%$ confidence limits in parentheses) alltrans retinoic acid $0.9\left(0.5^{-1} \cdot 5\right) \mu \mathrm{g}, \mathrm{I}_{3}$-cis retinoic acid $\mathrm{I} \cdot 3(\mathrm{I} \cdot 0-\mathrm{I} \cdot 6) \mu \mathrm{g}$, all-trans $\alpha$-retinoic acid, the carboxylic acid derivative of $\alpha$-retinol, $2 \cdot 4(2 \cdot 1-2 \cdot 8) \mu \mathrm{g}$, and $\gamma$ retinoic acid with an open chain instead of a ring, $12 \cdot 3(8 \cdot 7-17 \cdot 3) \mu \mathrm{g}$.

As a method for testing toxicity in compounds to be used on man, it is of less direct relevance than in vivo tests on rats and mice, but it is quick and cheap and is closer to the in vivo situation than most in vitro tests, and might serve to reduce the numbers of animals used for toxicity testing, an emotive issue nowadays.

Thus the study of chemical specificity and vitamin A activity has expanded from the early work designed to establish the features in a molecule necessary for restoring growth in vitamin $A$ deficient animals. The structural specificities for these other activities of vitamin A are so wide (see Bertram, 1980; Lotan et al. 1980; Newton et al. 1980; Trown et al. 1980) that they cannot be expressed except in terms of diffuse generalizations of slight predictive value. The profiles of activities of retinoids can vary substantially (Lotan et al. I 980; Trown et al. 1980) and we are handicapped by not knowing how they operate at the molecular level, and how effective a concentration can be maintained at the active site.

This flood of work on structure and vitamin A activity has largely passed by the function of vitamin $A$ in reproduction. Thompson et al. (1964) showed that rats could be maintained on a diet deficient in retinol, but containing retinoic acid. The animals grew well and were healthy (except for a deficiency of visual pigment) but in males spermatogenesis ceased, and pregnant females resorbed their foetuses. Two possible explanations are that the structural specificity for the function of vitamin $A$ in reproduction differs from that for its systemic role, or that retinoic acid does not penetrate to the necessary site of action. Evidence can be assembled against either hypothesis.

It seems implausible that retinoic acid does not support spermatogenesis in rats because it fails to reach anywhere in the testis. Very large doses of retinoic acid are ineffective (Thompson et al. 1964), as are direct injections into the testis (Ahluwalia \& Bieri, 1971).

The inability of $\alpha$-retinol to support spermatogenesis (Sneider et al. 1974) could, however, be due to its not being transported by plasma RBP, as discussed earlier. Overriding the body's normal transport system by delivering retinoids artificially 
into organ cultures revealed that many retinoids showed unanticipated activity at the cellular level. Could the same apply to reproduction? This is impossible to test in organ cultures as it has not yet proved practicable to maintain spermatogenesis in vitro.

We decided to try instead analogous experiments by adopting the technique of Ahluwalia \& Bieri (197I) of injecting compounds directly into the rat testis. Border \& Pitt ( 1983 ) confirmed that small injections of retinol and retinyl acetate would restore spermatogenesis, as would retinaldehyde, presumably as a consequence of its reduction to retinol. Most other substances injected were ineffective, including retinoic acid (thus confirming the finding of Ahluwalia \& Bieri, 1971), $\alpha$-retinyl acetate, 3-dehydroretinol (vitamin $A_{2}$ ), 5,6-epoxyretinaldehyde, and 4oxoretinaldehyde. Retinaldehyde oxime (Fig. I, $\mathrm{R}=\mathrm{CH}=\mathrm{NOH}$ ) had a little activity when given in large doses (Border \& Pitt, 1983).

These results have an old-fashioned look. We seem to be back to the narrow view of vitamin A specin̂city gained from rat growth tests. The wider specificity seen for the anti-keratinizing action of the vitamin, after removal of the constraints of the absorption and transport systems, appears not to apply to that for the restoration of spermatogenesis in rats. If these results are taken at their face value, they imply that the structural specificity for spermatogenesis in the rat is very much narrower than for the anti-keratinizing action of the vitamin.

Injected retinaldehyde oxime had a little activity and it was possible to restore normal spermatogenesis by giving retinaldehyde oxime orally over a period of 10 weeks; no retinol but only retinaldehyde oxime could be found in the testis (Border \& Pitt, 1983). Tosukhowong \& Olson (1978) have found that 15 -methylretinol (Fig. I, $\mathrm{R}:=\mathrm{C}\left(\mathrm{CH}_{3}\right) \mathrm{HOH}$ ) given orally will support spermatogenesis. These findings, taken with those of Thompson et al. (1964), imply that a hydroxyl group is necessary at the end of the chain to support spermatogenesis in rats.

What seemed surprising was that no compound with a modified $\beta$-ionone ring was effective, in sharp contrast to what has been found for other actions of vitamin A. It is implausible on a priori evolutionary arguments that the structural requirements for spermatogenesis would be massively different from those for the anti-keratinizing action of the vitamin. During evolution the retinoids were first taken into the animal kingdom in the form of retinaldehyde, presumably formed by oxidative cleavage of carotenoids, to serve as the chromophoric group of the visual pigments. Insects, arthropods and cephalopods use retinaldehyde in their visual pigments but apparently need vitamin A for nothing else. It is only in the vertebrates that vitamin $A$ is essential for life. Vertebrates use retinaldehyde in their visual pigments, but have also taken its reduction product, retinol, for some still inadequately defined function or functions necessary for life. It seems unlikely that mammals have seized upon retinol for a different role in spermatogenesis. But why do injections of $\alpha$-retinol and 3 -dehydroretinol into rat testis not restore spermatogenesis?

It may be that our delivery system is too crude, and that the injected $3^{-}$ dehydroretinol and $\alpha$-retinol cannot get to the requisite operational site, even 
though they are well taken up by the intracellular CRBP (Ong \& Chytil, 1975). This is an objection hard to counter, as it is uncertain where retinol works in spermatogenesis after it has been taken up by testicular interstitial cells (McGuire et al. $198 \mathrm{I}$ ). If it is valid, it introduces a further element of specificity that is not seen in the tracheal organ cultures used by Sporn and co-workers, possibly involving the cell-surface receptor for plasma RBP reported by Bhat \& Cama (1979).

Perhaps the most surprising finding was the inability of injected $3^{-}$ dehydroretinol to promote spermatogenesis. 3 -Dehydroretinol has nearly half the activity of retinol in rat growth (Shantz \& Brinkman, 1950; Weiser, 1964). Furthermore, when given orally it restores spermatogenesis in rats maintained on retinoic acid (Howell et al. 1967 ). But it did not promote spermatogenesis when two injections, each of $250 \mu \mathrm{g}$, were given - even though it will bind to CRBP (Ong \& Chytil, 1975).

Border \& Pitt (198I) reported that in the testes of rats maintained on retinoic acid plus small oral doses of 3 -dehydroretinol for 19 weeks, only retinol could be detected, indicating that 3 -dehydroretinol was being converted to retinol to function in spermatogenesis. The interpretation of the results of this experiment is not unequivocal, as the possibility exists that 3 -dehydroretinol might have been exerting a sparing action on the small amounts of retinol that seem always to be present in the tissues of animals maintained on retinoic acid. For example, T. E. $\mathrm{M}$. Wilson (unpublished work) has found $\mathrm{I} 3$ and $40 \mu \mathrm{g}$ retinol/g liver and kidney respectively in a rat that had actually died from vitamin A deficiency after previously having been maintained on retinoic acid for 18 months.

There are indications in the work of Cama et al. (1952) and Yoshikami et al. ( 1969 ) that rats can convert some 3 -dehydroretinol to retinol but their results too could arise from sparing action.

If 3 -dehydroretinol does not work as such, we would have the paradoxical situation of having on the one hand results in organ culture revealing a vastly wider range of structures capable of discharging the action of vitamin A in cells, and on the other of work with intact animals eliminating the only exception to the rule that an unmodified $\beta$-ionone ring is necessary for substantial activity in vivo.

The big revival of interest in the relationship between chemical structure and vitamin A activity has stemmed from the realization that vitamin A activity covers much wider phenomena than the prevention or cure of deficiency in intact animals.

The extension of the concept of vitamin A activity has been marked by an ever greater expansion of the variety of chemical structures manifesting such activities. The relatively simple generalizations of some years ago (see, for example, Pitt, 1965) are now seen to be the consequences of the constraints imposed by working with intact animals. In a strictly nutritional sense these limitations are realistic and the conclusions still valid.

The recognition of the wide diversity of structures capable of manifesting vitamin $A$ activity stems from the use of experimental procedures that can override, to some extent, the mechanisms of an animal body normally 
discriminating against substances other than retinol. Structural specificities have consequently become more nebulous and difficult to describe succinctly. The enlarged range of active retinoids does, however, offer a hopeful prospect as vitamin $A$ expands from nutrition into pharmacology.

\section{REFERENCES}

Ahluwalia, B. \& Bieri, J. G. (1971). J. Nutr. 101, 141.

Ames, S. R. (1965). Fedn Proc. Fedn Am. Socs exp. Biol. 24, 917.

Bertram, J. S. (1980). Cancer Res. 40, $314 \mathrm{I}$.

Bhat, M. K. \& Cama, H. R. (1979). Biochim. biophys. Acta 587, 273.

Blatz, P. E., Lin, M., Balasubramaniyan, P., Balasubramaniyan, V. \& Dewhurst, P. B. (I969). f. Am. chem. Soc. 91, 5930 .

Bollag, W. \& Matter, A. (1981). Ann. N.Y. Acad. Sci. 359, 9.

Border, P. M. \& Pitt, G. A. J. (1981). Int. Congr. Nutr. XII, 4 I (abstracts).

Border, P. M. \& Pitt, G. A. J. (I983). Proc. Nutr. Soc. 42, 7A.

Cama, H. R., Dalvi, P. D., Morton, R. A. \& Salah, M. K. (1952). Biochem. F. 52, 542.

Chen, C. C. \& Heller, J. (1977). F. biol. Chem. 252, 5216.

Chytil, F. \& Ong, D. E. (1 978). Vitam. Horm. 36, I.

Clamon, G. H., Sporn, M. B., Smith, J. M. \& Henderson, W. M. (1975). Y. Nutr. 105, 215.

Clamon, G. H., Sporn, M. B., Smith, J. M. \& Saffiotti, V. (1974). Nature, Lond. $250,64$.

Cogan, U., Kopelman, M., Mokady, S. \& Shinitzky, M. (1976). Eur. F. Biochem. 65, 7 1.

Crouch, R., Katz, S., Nakanishi, K., Gawinowicz, M. A. \& Balogh-Nair, V. (1981). Photochem. Photobiol. 33, 9I.

Dowling, J. E. \& Wald, G. (1960). Proc. Nat. Acad. Sci. USA 46, 587.

Gomez, E. C. (198I). In Retinoids, p. 213 [C. E. Orfanos, O. Braun-Falco, E. M. Farber, C. Grupper, M. K. Polano and R. Schuppli, editors]. Berlin: Springer.

Goodman, D. S. \& Raz, A. (1972). F. Lipid Res. 13, 338.

Goodman, D. S., Smith, J. E., Hembry, R. M. \& Dingle, J. T. (1974). Y. Lipid Res. 15, 406.

Harris, P. H. (1 960). Vitom. Horm. 18, 34 I.

Hicks, R. M. (1983). Proc. Nutr. Soc. 42,83.

Horwitz, J. \& Heller, J. (1973). F. biol. Chem. 248, 6317.

Horwitz, J. \& Heller, J. (1974). F. biol. Chem. 249, 47 I 2.

Houghton, S. E. (1969). $\alpha$-Retinol. PhD Thesis, University of Liverpool.

Howell, J. McC., Thompson, J. N. \& Pitt, G. A. J. (1967). Br. F. Nutr. 2 1, 373.

Hughes, D. R. (1975). Aspects of the toxicity of retinoic acid. PhD Thesis, University of Liverpool.

Lotan, R. (1980). Biochim. biophys. Acta 605, 33 .

Lotan, R., Neumann, G. \& Lotan, D. (1980). Cancer Res. 40, 1097.

McGuire, B. W., Orgebin-Crist, M. C. \& Chytil, F. (1981). Endocrinology, ro8, $65^{8}$.

Morgan, B., Thompson, J. N. \& Pitt, G. A. J. (1969). Br. J. Nutr. 23, 899.

Morton, R. A. \& Pitt, G. A. J. (1969). Adv. Enzymol. 32, 97.

Muhilal, H. \& Glover, J. (1975). Biochem. Soc. Trans. 3, 744.

Newton, D. L., Henderson, W. R. \& Sporn, M. B. (1980). Cancer Res. 40, $34 \mathrm{I} 3$.

Ong, D. E. \& Chytil, F. (1975). Nature, Lond. 255, 74.

Peck, G. L. (1980). Archs Dermatol. 1 16, 283.

Peck, G. L., Olsen, T. G., Yoder, F. W., Strauss, J. S., Downing, D. T., Pandya, M., Butkus, D. \& Araud-Battandier, J. (1979). New Engl. F. Med. 300, 329 .

Pitt, G. A. J. (1965). Proc. Nutr. Soc. 24, I 53.

Pitt, G. A. J. (1969). Am. F. clin. Nutr. 22, 1045.

Pitt, G. A. J. (1978). Wld Rev. Nutr. Diet. 31, 65.

Rask, L., Anundi, H., Böhme, J., Eriksson, U., Ronne, H., Sege, K. \& Peterson, P. A. (1981). Ann. N.Y. Acad. Sci. 359, 79.

Rask, L., Geijer, C., Bill, A. \& Peterson, P. A. (1980). Exp. Eye Res. 31, 201. 
Shantz, E. M. \& Brinkman, J. H. (1950). F. biol. Chem. 183, 467.

Smith, J. E., Milch, P. O., Muto, Y. \& Goodman, D. S. (1973). Biochem. F. 132, 82 1.

Sneider, W. D., Rosso, G. C., Rogers, A. E., Wolf, G., Dowling, J. E. \& Callahan, M. J. (1974). 7. Nutr. 104, 1662 .

Sporn, M. B., Clamon, G. H., Dunlop, N. M., Newton, D. L., Smith, J. M. \& Saffiotti, U. (1975). Nature, Lond. 253, 47.

Sporn, M. B., Dunlop, N. M., Newton, D. L. \& Henderson, W. R. (1976). Nature, Lond. 263, IIO.

Sporn, M. B., Dunlop, N. M., Newton, D. L. \& Smith, J. M. (1976). Fedn Proc. Fedn Am. Socs exp. Biol. 35, 1332.

Thompson, J. N., Howell, J. McC. \& Pitt, G. A. J. (1964). Proc. Roy. Soc. B159, 510.

Thompson, J. N., Howell, J. McC., Pitt, G. A. J. \& McLaughlin, C. I. (I969). Br. F. Nutr. 23, 47 1.

Thompson, J. N. \& Pitt, G. A. J. (1960). Nature, Lond. 188, 672.

Tosukhowong, P. \& Olson, J. A. (1978). Biochim. biophys. Acta 529, $43^{8}$.

Trown, P. W., Palleroni, A. V., Bohoslawec, O., Richelo, B. N., Halpern, J. M., Gizzi, N., Geiger, R., Lewinski, C., Machlin, L. J., Jetten, A. \& Jetten, M. E. R. (1980). Cancer Res. 40, 212 .

Tsambaos, D. \& Orfanos, C. E. (1981). Pharmac, Ther. 14, 355.

Turton, J. A., Hicks, R. M., Gwynne, J., Hunt, R., Palmer, L., Medawar, P. B. \& Hawkey, C. M. (1983). Proc. Nutr. Soc. 42, I A A.

Weiser, H. (1964). Biochem. biophys. Res. Commun. 14, 183.

Willetts, S. R., Houghton, S. E., Jones, G. \& Pitt, G. A. J. (1976). Proc. Nutr. Soc. 35, 142 A.

Yoshikami, S., Pearlman, J. T. \& Crescitelli, F. (1969). Vision Res. 9, 633.

Zile, M. \& DeLuca, H. F. (1968). Y. Nutr. 94, 302. 